\title{
Testosterone amendment alters metabolite profiles of the soil microbial community ${ }^{\text {is }}$
}

\author{
Jing Ding a, Hongwei Sun ${ }^{\mathrm{a}}$, Aiping Liang a , Jin Liu ${ }^{\mathrm{a}}$, Lehui Song ${ }^{\mathrm{c}}$, Min Lv ${ }^{\mathrm{c}}$, Dong Zhu ${ }^{\mathrm{b}, *}$ \\ a School of Environmental and Material Engineering, Yantai University, 30 Qingquan Road, Yantai, 264005, China \\ b State Key Laboratory of Urban and Regional Ecology, Research Centre for Eco-Environmental Sciences, Chinese Academy of Sciences, 18 Shuangqing Road, \\ Beijing, 100085, China \\ c CAS Key Laboratory of Coastal Environmental Processes and Ecological Remediation, Yantai Institute of Coastal Zone Research, Chinese Academy of \\ Sciences, 17 Chunhui Road, Yantai, 264003, China
}

\section{A R T I C L E I N F O}

\section{Article history:}

Received 17 August 2020

Received in revised form

23 September 2020

Accepted 24 October 2020

Available online 26 October 2020

\section{Keywords:}

Hormones

Contaminant

Metabolomics

Bacterial community

\begin{abstract}
A B S T R A C T
Steroid hormones are prevalent in the environment and have become emerging pollutants, but little is known about their effects on soil microbial community composition and function. In the present study, three representative soils in China were amended with environmentally relevant concentrations of testosterone and responses of soil bacterial community composition and soil function were assessed using high-throughput sequencing and nontargeted metabolomics. Our results showed that testosterone exposure significantly shifted bacterial community structure and metabolic profiles in soils at Ningbo (NB) and Kunming (KM), which may reflect high bioavailability of the hormone. Abundances of several bacterial taxa associated with nutrient cycling were reduced by testosterone and metabolites related to amino acid metabolism were downregulated. A close connection between bacterial taxa and specific metabolites was observed and confirmed by Procrustes tests and a co-occurrence network. These results provide an insight into the effects of steroid hormones on soil microbial community and highlight that nontargeted metabolomics is an effective tool for investigating the impacts of pollutants.
\end{abstract}

(C) 2020 Elsevier Ltd. All rights reserved.

\section{Introduction}

The steroid hormones are phylogenetically conserved but are newly prevalent in the environment (Alon et al., 2007; Kolpin et al., 2002). Human and animal wastes are a major source of hormones in the environments (Casey et al., 2004; Jarvinen et al., 2020). Naturally produced hormones are found in human and livestock waste, and synthetic hormones are found in manure (Casey et al., 2004). The concentrations can be nearly $100 \mu \mathrm{g} \mathrm{kg}^{-1}$ in manure (Goeppert et al., 2014; Havens et al., 2020). Manure and sewage sludge are widely used as agricultural fertilizers, leading to the entry of these compounds into soil ecosystems (Arnon et al., 2008; Stumpe and Marschner, 2007). The long term application of agricultural fertilizers has increased hormone concentrations by more than 10-fold compared with unfertilized soils (Casey et al., 2004). Although hormones are trace pollutants in soil (Goeppert et al.,

\footnotetext{
\& This paper has been recommended for acceptance by Dr. Yong Sik Ok.

* Corresponding author.

E-mail address: dongzhu@rcees.ac.cn (D. Zhu).
}

2014; Havens et al., 2020), their ecological impacts on soil should not be ignored considering that hormones are active at low concentrations (Barbosa et al., 2008).

Primary focus on steroid hormones has been concentrated on their estrogenic effects. More recently, the androgenic and antiandrogenic activities have been reported in aquatic environment. Naturally occurring reproductive androgenic hormones, such as testosterone, which is often used in endocrine therapy, are commonly detected in different environmental media (Ewing et al., 1977). Long-term exposure to testosterone can induce a decrease in fecundity and fertility for the Daphnis, though the acute toxicity of testosterone is not obvious (Barbosa et al., 2008). Testosterone, stable and highly lipophilic in soils, exists mainly through adsorption onto soil particles and organic matter (Ma et al., 2015; Qi and Zhang, 2016a, b) and is a dominant androgen in soil ecosystems (Havens et al., 2020; Qi and Zhang, 2016b), indicating a potential impact on nontargeted soil species. The negative impact of testosterone on soil methane-oxidizing bacteria has been reported (Ruan et al., 2014). Considering most studies on ecological effects were concentrated in aquatic environments (Barbosa et al., 2008), influences of steroid hormones on soil microbes should not be 
neglected. However, there is a knowledge gap for the impacts of testosterone on soil microbial community structure and function.

Soil provides many essential ecosystem services, including nutrient cycling, food supply, and climate regulation (Bunemann et al., 2018). Thus, investigating changes in soil quality and biological function caused by soil contaminants such as testosterone is essential, which shows high biological activity at low concentrations. The use of "omic"-based technologies, including genomics, transcriptomics, proteomics, and metabolomics, offers new means to evaluate biological function (Withers et al., 2020). Functional shifts at the molecular level are more sensitive than traditional indicators and may provide deeper insight into biological responses to environmental stress (Pagano et al., 2016). Moreover, metabolomics represent the final "omic" level of functional entities and integrate response at gene, transcription, and translation levels. Soil metabolites have been suggested to be an effective way to evaluate changes in soil quality and function since they reflect important metabolic pathways for soil microbial communities (Withers et al., 2020), and metabolites have been measured in a few studies to describe the toxicity of nanoparticles on plants and soil animals ( $\mathrm{He}$ et al., 2020; Li et al., 2020). Hence, soil metabolites might provide a holistic understanding of soil function responses to environmental contaminants.

In the present study, to examine the impact of steroid hormones on soil microbial community composition and function, three types of representative soils in China were amended with environmentally relevant concentrations of testosterone. Nontargeted metabolites, of which hundreds will be detected, were used to characterize soil functional alterations, which could also facilitate the investigation of relationships between soil microbial community and soil metabolites.

\section{Materials and methods}

\subsection{Soil sampling and treatment}

Three agricultural soil types that were collected from Changchun (CC, Jilin province), Ningbo (NB, Zhejiang province), and Kunming (KM, Yunnan province) were black soil, fluvo-aquic, and laterite, respectively. The basic properties of sites and soils are provided in supplemental information (Table S1). Organic fertilizers were not applied to these soils in the last five years. The top $20 \mathrm{~cm}$ soil was air-dried and sieved to $2 \mathrm{~mm}$. A total of $0.8 \mathrm{~mL}$ testosterone stock solution (15 $\mathrm{mg} \mathrm{L}^{-1}$ ) was mixed with $29.2 \mathrm{~mL}$ deionized water and then was equally sprinkled to each type of soil (200 g dry soil) using injector, and homogenized with hand (Li et al., 2019). The final concentration of testosterone was $60 \mu \mathrm{g} \mathrm{kg}^{-1}$, representing an environmentally relevant concentration (Casey et al., 2004). For each soil type, $200 \mathrm{~g}$ of untreated soil was used as a control. As ethanol was used to prepare testosterone stock solution, the controls were amended with the same concentration and amount of ethanol solution. The water content of the treated soils was equal to $55-65 \%$ of maximum water capacity. Then, $50 \mathrm{~g}$ of the moist soil was added to $100 \mathrm{~mL}$ beakers (in volume). Each treatment was kept static in the dark at $20{ }^{\circ} \mathrm{C} \pm 1{ }^{\circ} \mathrm{C}$ for four weeks. Water content was adjusted twice a week using weight balance method, and each treatment was performed in quadruplicate. At the end of the incubation, the soil sample in each beaker was homogenized again using hand. After that, $10 \mathrm{~g}$ moist soil was collected using the method of coning and quartering. The sampling sites for the soil from Changchun, Ningbo, and Kunming were abbreviated to CC, NB, and KM, respectively. The label CK and GT represented the treatment without and with testosterone amendment, respectively.

\subsection{DNA extraction, sequencing, and microbial community analysis}

Bacterial 16S rRNA gene high-throughput sequencing on an Illumina HiSeq 2500 platform (Novogene, Tianjin, China) was conducted to evaluate soil bacterial communities. The detailed protocol was described in our previous study (Ding et al., 2019). In brief, DNA was extracted from $0.25 \mathrm{~g}$ of soil using DNA extraction kits (PowerSoil DNA Extraction kit, MO-BIO Laboratories). Barcoded primer pairs (515F and 806R) were selected to amplify the V4 region of $16 \mathrm{~S}$ rRNA gene using the following protocol: $95^{\circ} \mathrm{C}$ for $5 \mathrm{~min}$, followed by 30 cycles of $94^{\circ} \mathrm{C}$ for $45 \mathrm{~s}, 50^{\circ} \mathrm{C}$ for $45 \mathrm{~s}, 68^{\circ} \mathrm{C}$ for $45 \mathrm{~s}$, and a final step at $72{ }^{\circ} \mathrm{C}$ for $10 \mathrm{~min}$. The PCR products were purified and pooled in equimolar amounts before sequencing.

After an initial quality check, paired-end reads were processed with USEARCH version 10.240 (Edgar, 2013). Sequences were quality-filtered using a default "maxee" value of 1.0 , and unique sequences were denoized and de novo mapped into zero-radius operational taxonomic units (zotus) with the unoise 3 algorithm (Edgar, 2016). Zotus were identified using BLAST on the UNITE database, and identities of zotus were verified in the NCBI database (Vendl et al., 2019). Microbial alpha diversity was estimated with the Shannon index and Simpson index, calculated in R3.4.1. Microbial beta diversity was determined using the Bray-Curtis dissimilarity matrix. Adonis tests performed in R3.4.1 were used to assess differences in bacterial community structure among treatments.

\subsection{Soil nontargeted metabolomic detection and analysis}

Soil samples $\left(-80^{\circ} \mathrm{C}\right)$ were sent to the metabolomics analysis platform in Majorbio (Shanghai, China) for evaluation of nontargeted metabolomics. The detailed protocol was described in previous studies with slight adjustment (He et al., 2020). In brief, $1000 \mathrm{mg}$ soil aliquots were extracted with $1.3 \mathrm{~mL}$ of methanol/ water $(4: 1, \mathrm{v} / \mathrm{v})$ solution for $30 \mathrm{~min}$ at $4{ }^{\circ} \mathrm{C}$ in a vortex mixer. The extract was centrifuged at 13,000 $\mathrm{g}$ (relative centrifugal force) for $15 \mathrm{~min}$, and the supernatant was transferred. After three times of extraction, the supernatant were mixed and concentrated under nitrogen gas. Finally, samples were dissolved in $100 \mu \mathrm{L}$ and subjected to LC-MS/MS analysis.

Chromatographic separation of metabolites was performed on an ExionLCTMAD system (AB Sciex, USA) equipped with an ACQUITY UPLC BEH C18 column $(100 \times 2.1 \mathrm{~mm} ; 1.7 \mu \mathrm{m}$; Waters, Milford, USA). Mobile phases consisted of $0.1 \%$ formic acid in water and $0.1 \%$ formic acid in acetonitrile/isopropanol $(1: 1, \mathrm{v} / \mathrm{v})$. The UPLC system was coupled to a quadrupole time-of-flight mass spectrometer (Triple TOFTM5600+, AB Sciex, USA) equipped with an electrospray ionization source operating in positive and negative mode.

A pooled quality control (QC) sample was prepared by mixing equal volumes of all samples for system conditioning and QC. QC samples were prepared and tested in the same manner as analytic samples. The control represented the entire samples set and was injected after every ten samples to monitor analysis stability.

Data were analyzed with online analysis platform (Majorbio, Shanghai, China) following online instructions. Data were normalized with Pareto scaling and log-transformed before deep analysis. Principal component analysis (PCA) using data collected from positive and negative ion mode, separately, was used to reduce the complexity of the data matrix and summarized differences among groups. A supervised clustering method - partial least squares-discriminant analysis (PLS-DA) - was employed to maximize variation among groups and distinguish variables useful for class separation. Variable importance in projection (VIP) values were calculated to show the contribution to distinction in the PLS- 
DA model. Metabolites with VIP value $>1.0$ and $p$-value $<0.05(t-$ test) were the criteria used to identify variable significantly affected by testosterone exposure (Zhang et al., 2020). A heatmap that combined hierarchical clustering analysis was developed to show changes of discriminating metabolites after testosterone amendment. For pathway analysis of the identified metabolites, an online resource (MetaboAnalyst 4.0, http://www.metaboanalyst.ca/) was used, and a $p$-value $<0.05$ for enrichment analysis and an impact value $>0.1$ for pathway topology analysis were set as threshold values (Xia and Wishart, 2010). Differences in metabolome between control and testosterone treatment for different soil types were compared separately.

\subsection{Correlation analysis between soil microbial community and} soil metabolite profiles

Procrustes and Mantel tests and co-occurrence network were used to investigate correlations between soil microbial community structure and soil metabolite profiles. Significance of Spearman's correlation coefficients $\left(\mathrm{R}^{2}\right)$ in the co-occurrence network were $>0.8$ and $p<0.01$ since these measures display statistical correlation. All analysis was completed in R 3.4.1 platform.
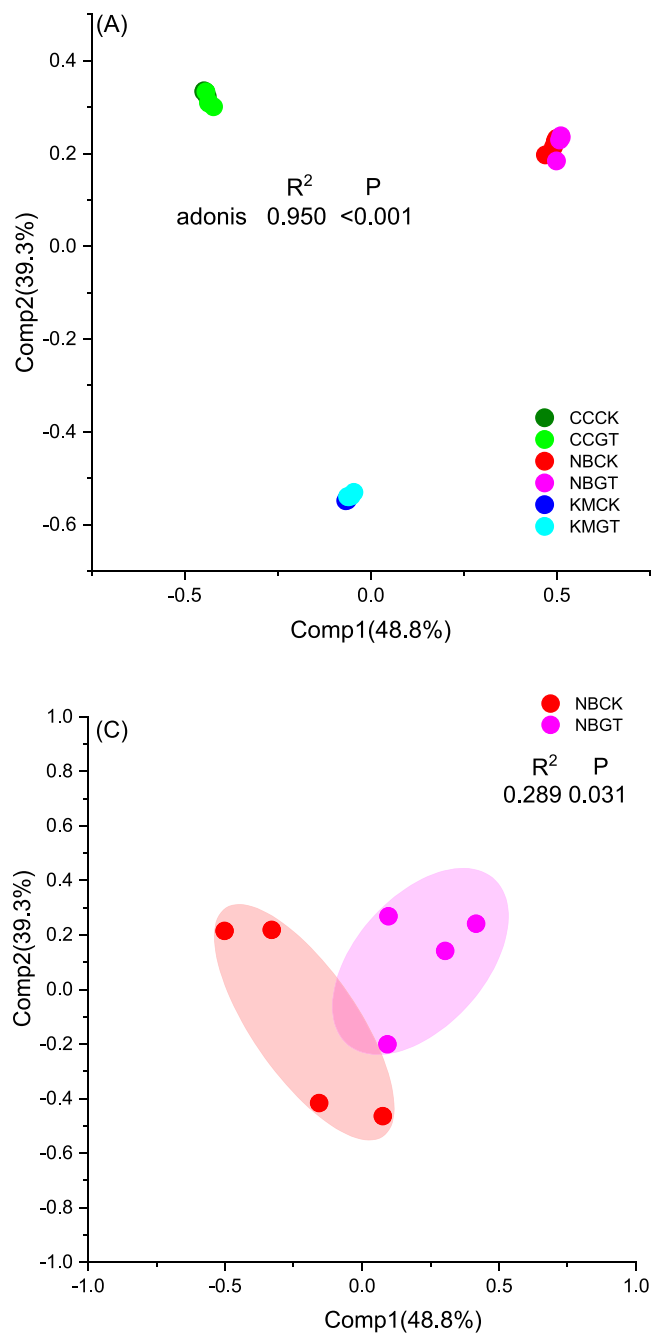

\section{Results and discussion}

\subsection{Testosterone amendment altered soil microbial community composition}

High-throughput bacterial 16S rRNA gene sequencing was used to investigate the responses of soil microbial community composition to testosterone amendment. PCoA showed soil microbial communities were clustered by soil type (Fig. 1A). This result was confirmed by Adonis analysis $(p<0.001)$. Thus, initial microbial communities differed by soil type. The finding is consistent with significant differences in physical-chemical properties of different soil types (Table S1, $p<0.05$ ), and that soil type is a determinant of microbial communities (Table S2; Bahram et al., 2018). Additionally, PCoA results showed that testosterone amendment changed microbial community composition in fluvo-aquic of NB and laterite of $\mathrm{KM}$, according to Adonis test results (Fig. 1C, D, $p<0.05$ ). However, no significant difference in the microbial community was observed between testosterone and control groups from the black soil of CC (Fig. $1 \mathrm{~B}, p=0.05$ ). Thus, the responses of microbial community to testosterone amendment varied depending on soil types (Table S2). The higher soil organic carbon content from CC (black soil) than
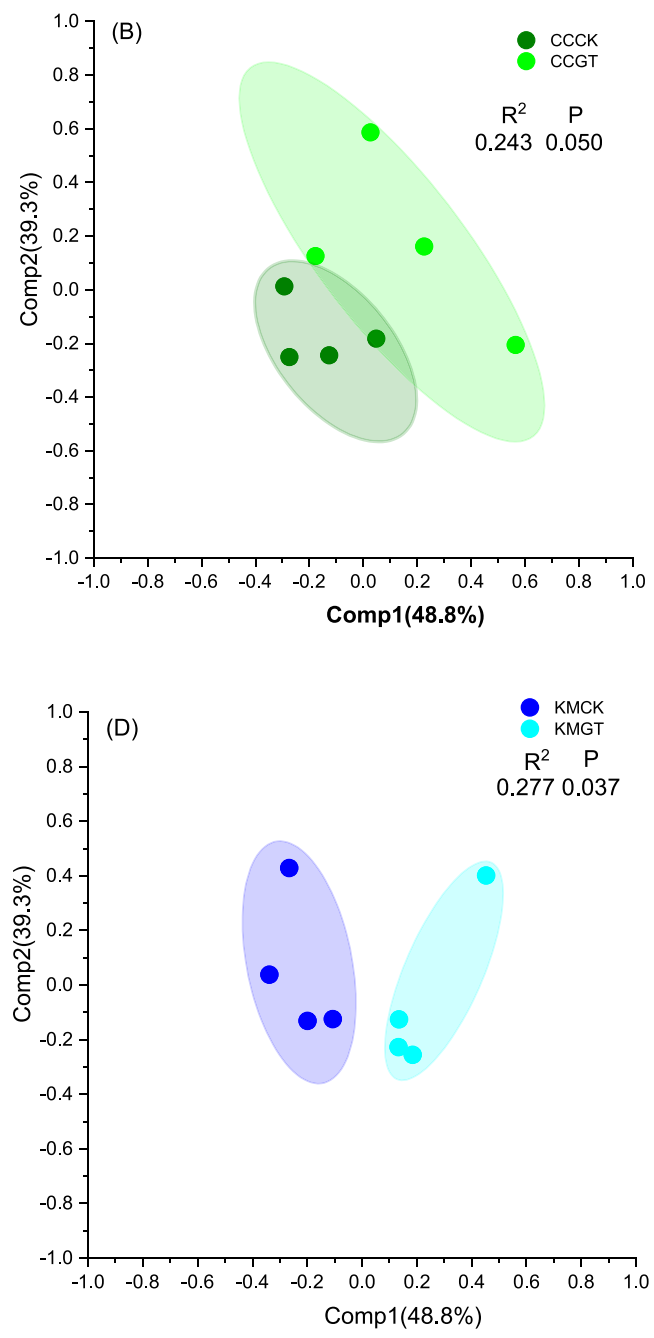

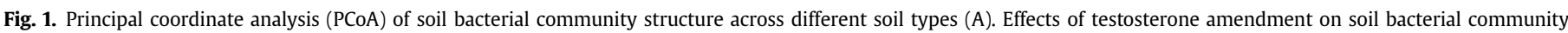

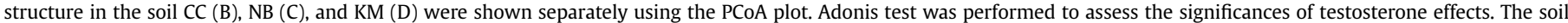

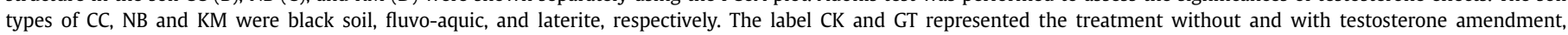
respectively. 

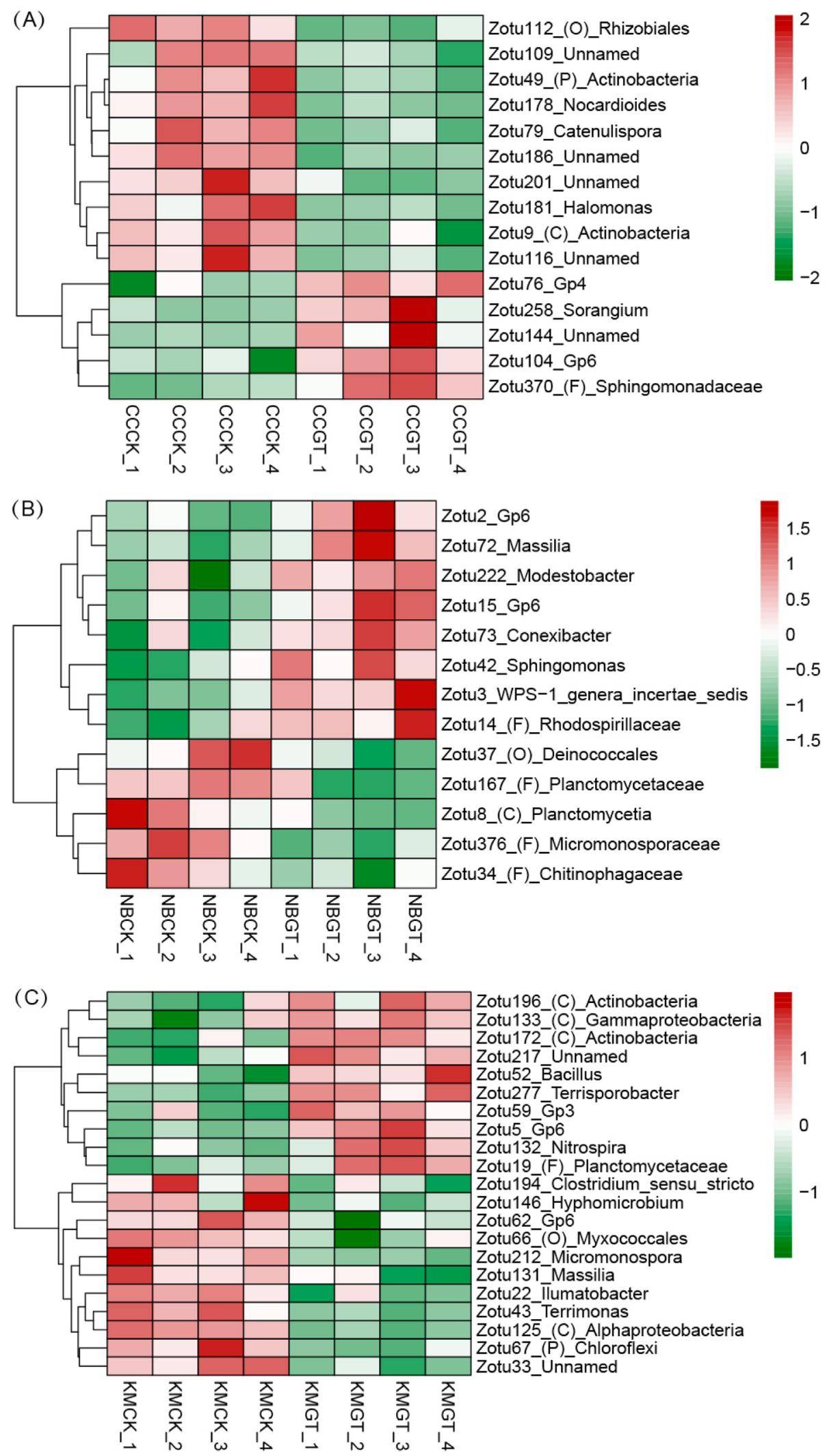

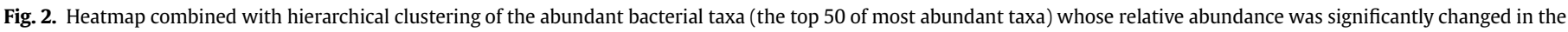

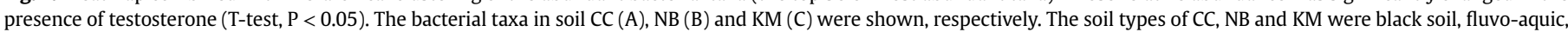
and laterite, respectively. The label CK and GT represented the treatment without and with testosterone amendment, respectively. 

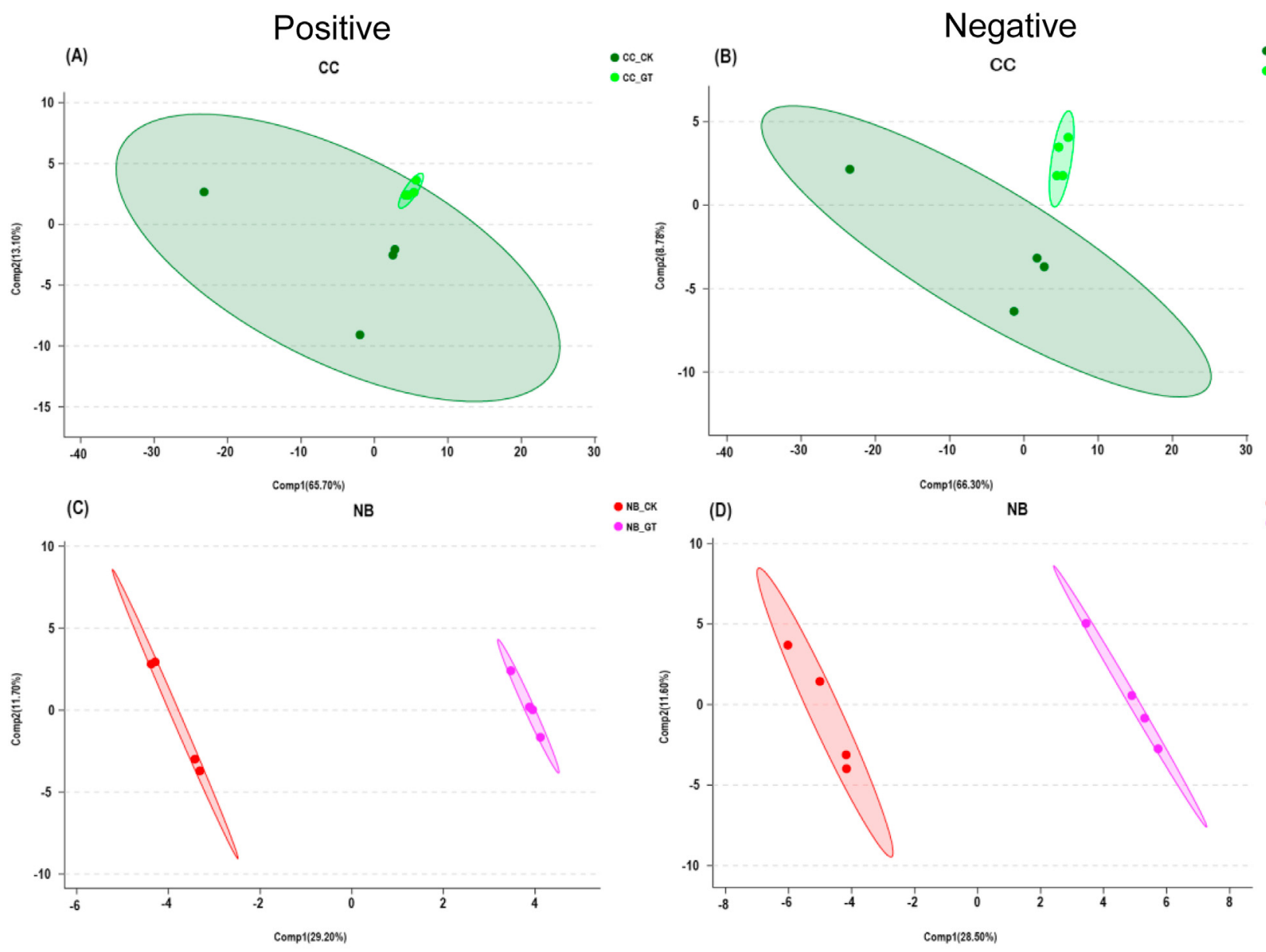

(E)

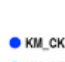

(F)

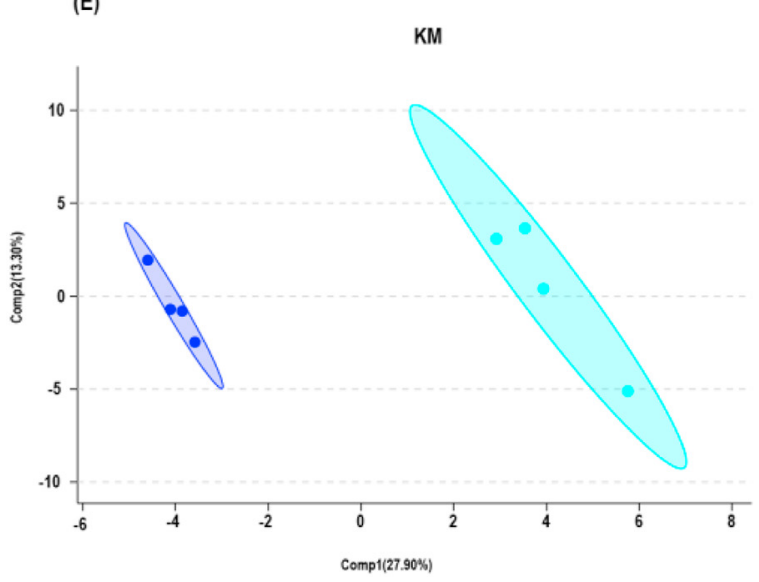

- ku_ck

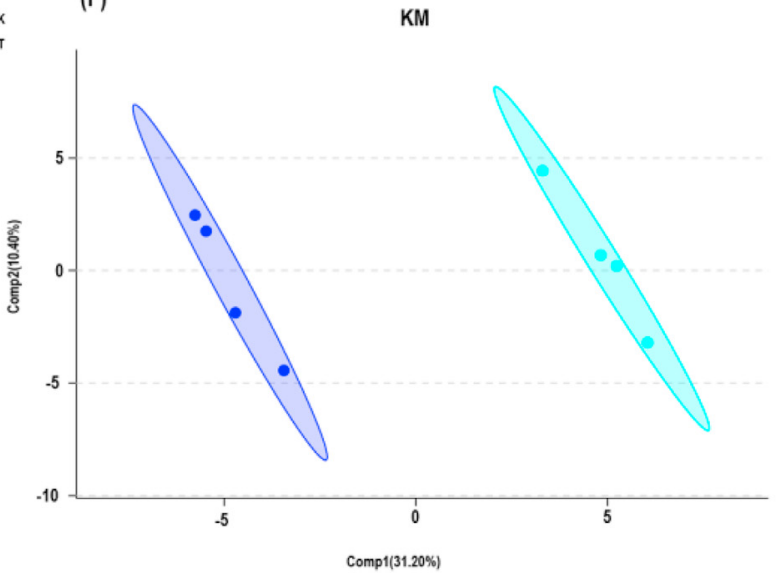

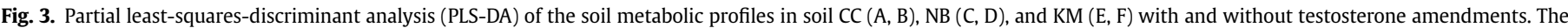

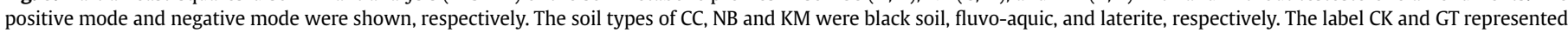
the treatment without and with testosterone amendment, respectively.

from NB (fluvo-aquic) and KM (laterite) might partly explain the variable responses in different soil types (Table S1), since testosterone exists in soil mainly through adsorption to soil organic matter, and bioavailability may be low in soil with high organic matter content (Ma et al., 2015; Qi and Zhang, 2016a). Besides, the close connections between $\mathrm{pH}$ and clay contents and soil microbial community indicated that these properties may also affect bioavailability of testosterone in soil, leading to the different responses of soil microbial community in different soil type to testosterone amendment.

Shannon index and Simpson index were used to assess changes in the diversity of soil microbial communities due to testosterone exposure, and the significant impacts with a decreased index were observed in NB soil (Table S3). Notably, the effects of testosterone on the alpha diversity were different among different soil types (Table S3). The varied environmental behavior of testosterone in different soil types such as the bioavailability might explain the type-specific responses. Generally, the microbial community with 
high diversity is resistant to stress (Allison, 2004). Therefore, the inconspicuous responses of the microbial community in soil from CC might also be due to their high community diversity.

Taxon-based analysis (in zotu level) indicated that several soil microbial taxa exhibited significant changes in abundance (Fig. 2). Based on the difference analysis of the 100 most abundant taxa, 15, 13 , and 21 taxa were significantly changed in CC, NB, and KM soils, respectively, in comparison to the corresponding control treatment (Fig. 2). It was noted that these abundant taxa were not the testosterone-degrading bacteria isolated from soils (Chiang et al., 2020). The impact of testosterone on soil microbial communities might be a main reason for the alterations. A few studies have examined the effects of steroid hormones on soil microbial community and function. For example, Ruan et al. (2014) found that testosterone pollution inhibited microbial methane oxidation in water columns and promoted discharge of methane from water (Ruan et al., 2014). Further, a number of microbes that play important roles in maintaining soil functions, such as carbon, nitrogen, and phosphorus cycling, were reduced by testosterone amendment (Fig. 2). For example, the relative abundance of Halomonas in CC soil and Deinococcales in NB soil decreased upon exposure to testosterone. Both organisms can mineralize soil phosphorus (Ragot et al., 2015; Ye et al., 2020). Moreover, Hyphomicrobium in KM soil was associated with methylotrophs and showed a decrease in relative abundance (Macey et al., 2020). Numerous studies reported negative effects of contaminants, including metals (heavy metal and metal nanoparticles) and organic components, on soil microorganisms related to nutrient cycling (Li et al., 2019; Zhang et al., 2020). In the present study, similar effects were also observed, indicating that contamination by testosterone might lead to an impact on soil eco-functions, even at low environmental concentrations.

\subsection{Testosterone amendment altered soil metabolic profiles}

A total of 3658 and 3875 metabolites in soils were determined in positive and negative ion mode, respectively, using LC-MS/MSbased nontargeted metabolomics. A total of 301 metabolites were identified and named based on the human metabolome database (HMDB) and Kyoto Encyclopedia of Genes and Genomes (KEGG) (Table S4). The PCA plot suggested varied metabolic profiles among different soil types, consistent with the results of soil microbial community structures. Hierarchical clustering using the top 100 identified metabolites (Fig. S2) confirmed this finding. Further, PLSDA mode selected the most predictive and discriminative features to assist classification. The loading plot from this analysis showed clear separation in metabolites between testosterone amended soils and controls for both fluvo-aquic of NB and laterite of KM (Fig. 3). However, this separation was only observed in negative ion mode for CC soils (Fig. 3B). The above results indicated that testosterone amendment significantly altered the soil metabolite profiles, and different soil types showed different responses. Exposure to metal nanoparticles and persistent organic pollutants significantly alters soil metabolites (Li et al., 2019; Zhang et al., 2020), and this study extended these observations to steroid hormones.

VIP in the PLS-DA model was calculated to examine the changes in soil metabolites in detail. Metabolites with VIP values $>1.0$ and $p$-values $<0.05$ (T-test) were considered significantly influenced by testosterone amendment. Twelve and seven significantly affected metabolites were identified in NB and KM soils, respectively; and the top 20 metabolites with highest VIP values were listed in Fig. 4. Not all metabolites with high VIP values were significantly altered in CC soil (Fig. 4A). This finding parallels the results of soil microbial community structure where NB and KM soils were more sensitive to testosterone amendment than CC soils (Fig. 1). The higher bioavailability of testosterone and lower microbial community diversity in fluvo-aquic of NB and laterite of KM might partly explain these responses. The most affected metabolites decreased upon testosterone amendment, except for a small number of unregulated compounds (Fig. 3 and Table S4), indicating that the impacts of testosterone on soil function may be negative, since soil metabolic profiles are expected to reflect soil functions (Withers et al., 2020). Generally, environmentally relevant concentration of testosterone can lead to changes in soil eco-functions, and the high sensitivity of soil metabolic profiles may better reflect these impacts induced by the emerging contaminants.

A number of amino acids and their precursors were significantly decreased by testosterone amendments, including L-isoleucine and L-phenylalanine in NB soil and L-glutamine and glutamyl-L-leucine in KM soil (Fig. 4), indicating that amino acid synthesis and metabolism in soil were apparently inhibited by testosterone. This was further confirmed by biochemical pathway analysis (Fig. S3). Two microbial metabolic pathways were significantly affected, including phenylalanine metabolism and phenylalanine, tyrosine, and tryptophan biosynthesis in NB and KM soil (Fig. S3). Since the soil amino acids are closely related to soil fertility and plant yield (Moreira and Moraes, 2017), their disorder due to testosterone amendment might be one mechanism for adverse effects on soil function (Zhao et al., 2019). Another metabolite notably decreased in soil metabolism was octadecadienoate in NB soil, suggesting that fatty acid metabolism was altered by testosterone (Fig. 4B). Since fatty acids, especially phospholipid fatty acids, are primary components of cell membranes (Quideau et al., 2016), such impacts could reflect the ROS overgeneration and abundance of microorganisms. Similarly, exposure to Ag nanoparticles significantly decreased several fatty acids in soil metabolites (Zhang et al., 2020). The present study suggests similar impact of testosterone on microbial cell membranes. Considering both amino acid and fatty acid metabolism are carbon- or nitrogen-related pathways, testosterone amendment clearly affected carbon and nitrogen metabolism in soil, which is consistent with bacterial community structure results.

\subsection{The close relationships between microbial communities and metabolites in soil}

Procrustes tests combined with Mantel tests correlated soil metabolic profiles with bacterial community structure (Fig. 5). Correlations were also observed between specific metabolites and bacterial community structure in NB and KM soils. Alterations in soil metabolic profiles were apparently mediated by soil microbial communities under testosterone stress (Fig. 5B and C), indicating altering certain metabolic pathways is a primary strategy for soil microbial communities to adapt to environmental stress. The close relationships are also observed in PAH-polluted soil (Li et al., 2019).

Co-occurrence network analysis further exhibited strong interrelationships of soil microbial taxa with soil metabolites in NB and KM soils and microbial taxa were the main driver for the changes in soil microbial metabolism (Fig. S4). For example, unidentified genera belonging to Actinobacteria in NB soil and genus Bacillus in KM soil co-occurred most frequently with several metabolites, including dihydroxyetiocholane, octadecadienoate, and alpha sanshool (Fig. S4; Table S5), and the positive co-occurrence indicated that metabolites might be compounds secreted by microbes. Negative co-occurrence might be due to specific microbial consumption or degradation (Devi et al., 2017). These findings indicated that soil metabolomics can be used to assess the adaptations of soil microbial communities to contaminants at the molecular level. 
(A) $\mathrm{CC}$
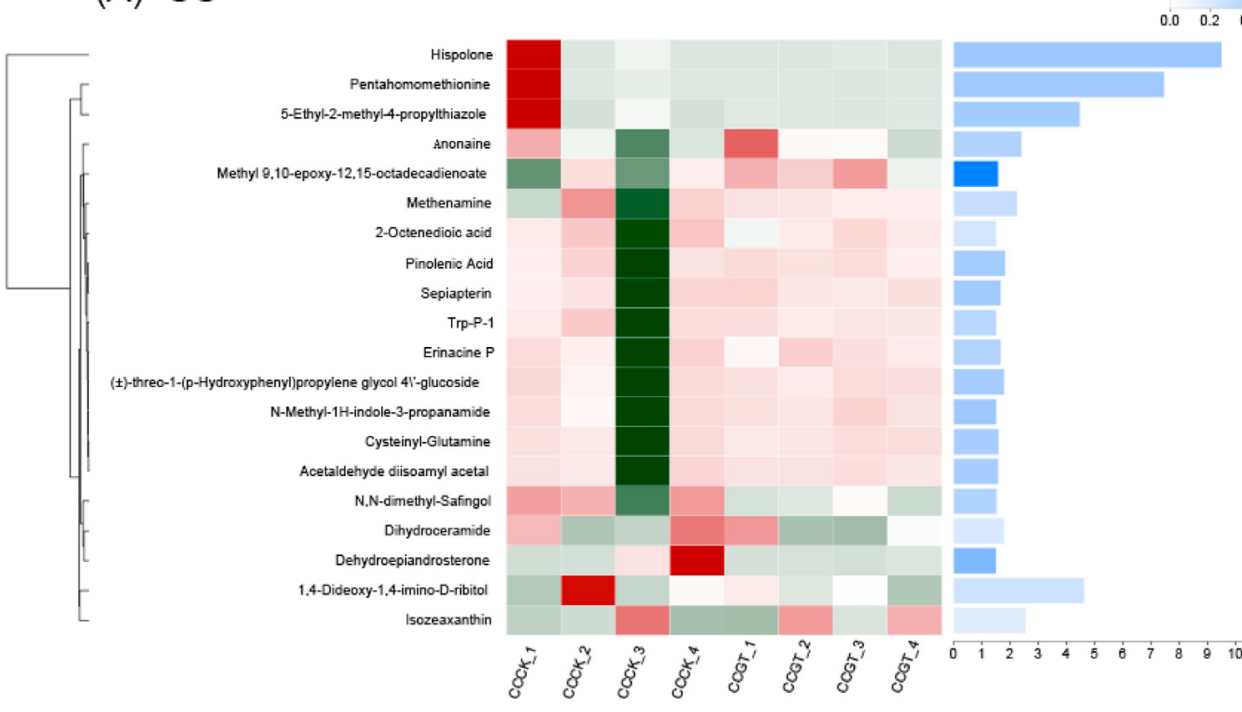

$\begin{array}{llllll}0.0 & 0.2 & 0.4 & 0.6 & 0.8 & 1.0\end{array}$

(B) NB
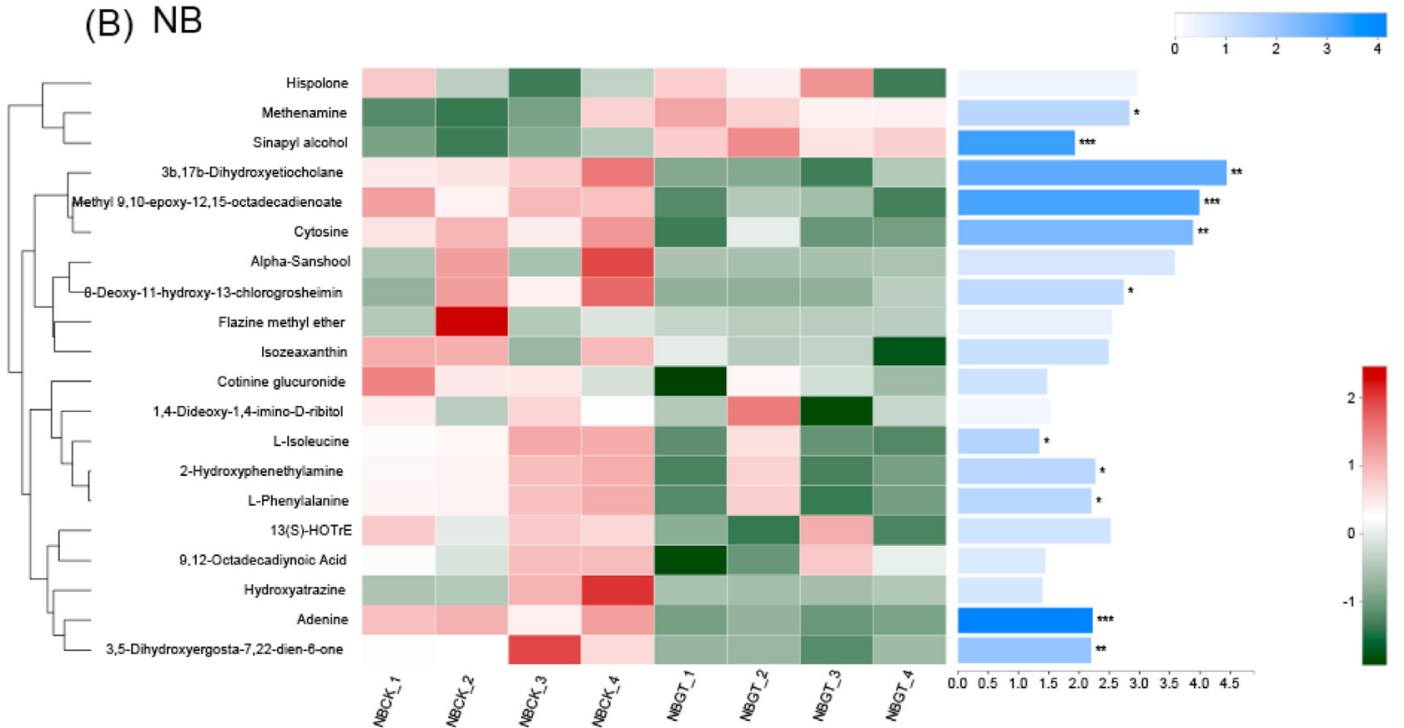

(C) KM
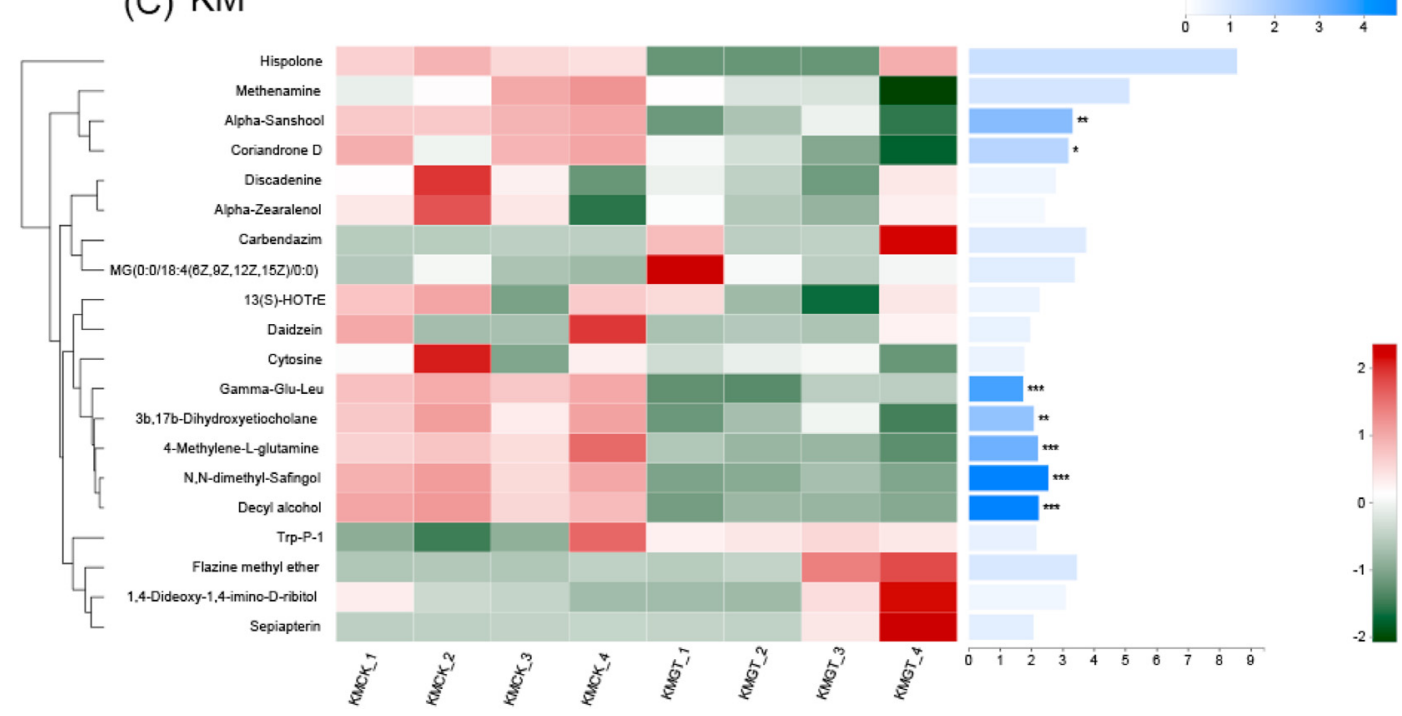

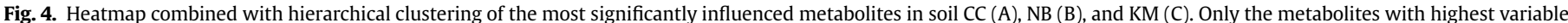

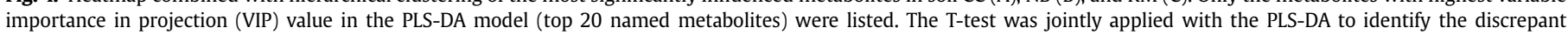

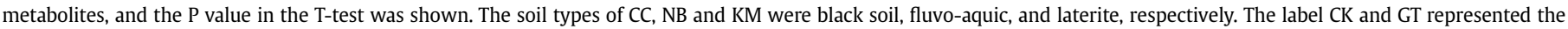
treatment without and with testosterone amendment, respectively. 

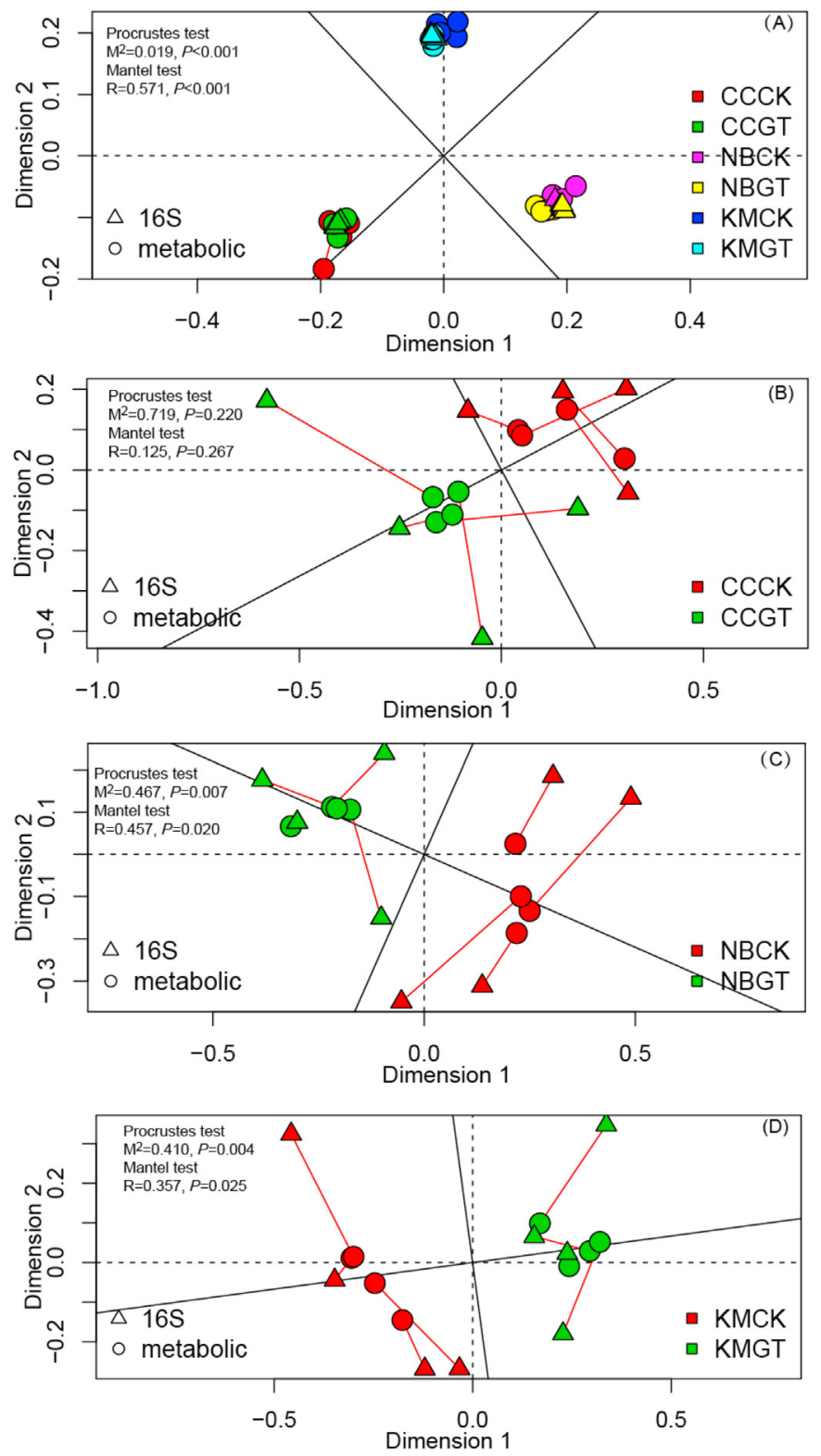

Fig. 5. Relationships between bacterial community structure and metabolic profiles (A) and discrepant metabolites (B, C, D) were revealed by Procrustes analysis and Mantel test. The label CK and GT represented the treatment without and with testosterone amendment, respectively.

\section{Conclusion}

The present study assessed the impacts of testosterone on microbial community structure and soil function of three representative soils in China using high-throughput sequencing and metabolomics. It turned out that testosterone shifted microbial community structure in NB and KM soils, but not in CC soil, indicating the effects of testosterone on soil microbiota may be typespecific. In addition, expression and metabolic processes of the soil metabolome were altered by testosterone amendment, illustrating the potential effects of testosterone on soil function. A close connection between bacterial community and metabolic profile in soil was also observed, suggesting a consistent response of them to environment stress. Therefore, the combination of soil microbiome and metabolome analysis can provide comprehensive insight into the ecological impact of the emerging contaminants.

\section{Author statement}

Jing Ding: Methodology, Investigation, Data curation, Funding acquisition, Writing - original draft. Hongwei Sun: Writing - review \& editing. Aiping Liang: Writing - review \& editing. Jin Liu: Writing - review \& editing. Lehui Song: Writing - review \& editing. Min Lv: Writing - review \& editing. Dong Zhu: Conceptualization, Writing review \& editing, Methodology, Data curation, Supervision.

\section{Declaration of competing interest}

The authors declare that they have no known competing financial interests or personal relationships that could have appeared to influence the work reported in this paper.

\section{Acknowledgments}

This study was financially supported by the National Natural Science Foundation of China (41807032 and 41601525), Major Scientific and Technological Innovation Projects of Key R\&D Programs in Shandong Province, China (2019JZZY020234) and Top Discipline in Materials Science of Shandong Province, China.

\section{Appendix A. Supplementary data}

Supplementary data to this article can be found online at https://doi.org/10.1016/j.envpol.2020.115928.

\section{References}

Allison, G., 2004. The influence of species diversity and stress intensity on community resistance and resilience. Ecol. Monogr. 74, 117-134.

Alon, G., Shore, L.S., Steinberger, Y., 2007. Correlation between levels of sex hormones (progesterone, testosterone, and estrogen) and ecophysiologicalbehavior stages in two species of desert snails (Sphincterochila zonata and Sphincterochila prophetarum) in the Northern Negev Desert. Gen. Comp. Endocrinol. 151, 122-127.

Arnon, S., Dahan, O., Elhanany, S., Cohen, K., Pankratov, I., Gross, A., Ronen, Z., Baram, S., Shore, L.S., 2008. Transport of testosterone and estrogen from dairyfarm waste lagoons to groundwater. Environ. Sci. Technol. 42, 5521-5526.

Bahram, M., Hildebrand, F., Forslund, S.K., Anderson, J.L., Soudzilovskaia, N.A., Bodegom, P.M., Bengtsson-Palme, J., Anslan, S., Coelho, L.P., Harend, H., HuertaCepas, J., Medema, M.H., Maltz, M.R., Mundra, S., Olsson, P.A., Pent, M., Polme, S., Sunagawa, S., Ryberg, M., Tedersoo, L., Bork, P., 2018. Structure and function of the global topsoil microbiome. Nature 560, 233-237.

Barbosa, I.R., Nogueira, A.J.A., Soares, A.M.V.M., 2008. Acute and chronic effects of testosterone and 4-hydroxyandrostenedione to the crustacean Daphnia magna. Ecotoxicol. Environ. Saf. 71, 757-764.

Bunemann, E.K., Bongiorno, G., Bai, Z., Creamer, R.E., De Deyn, G., de Goede, R., Fleskens, L., Geissen, V., Kuyper, T.W., Mader, P., Pulleman, M., Sukkel, W., van Groenigen, J.W., Brussaard, L., 2018. Soil quality - a critical review. Soil Biol. Biochem. 120, 105-125.

Casey, F.X.M., Hakk, H., Simunek, J., Larsen, G.L., 2004. Fate and transport of testosterone in agricultural soils. Environ. Sci. Technol. 38, 790-798.

Chiang, Y.R., Wei, S.T.S., Wang, P.H., Wu, P.H., Yu, C.P., 2020. Microbial degradation of steroid sex hormones: implications for environmental and ecological studies. Microb. Biotechnol. 13, 926-949.

Devi, S., Rehman, S.A.A., Tarique, K.F., Gourinath, S., 2017. Structural characterization and functional analysis of cystathionine beta-synthase: an enzyme involved in the reverse transsulfuration pathway of Bacillus anthracis. FEBS J. 284, 3862-3880.

Ding, J., Zhu, D., Hong, B., Wang, H.T., Li, G., Ma, Y.B., Tang, Y.T., Chen, Q.L., 2019. Long-term application of organic fertilization causes the accumulation of antibiotic resistome in earthworm gut microbiota. Environ. Int. 124, 145-152.

Edgar, R.C., 2013. UPARSE: highly accurate OTU sequences from microbial amplicon reads. Nat. Methods 10, 996-998.

Edgar, R.C., 2016. UNOISE2: Improved Error-Correction for Illumina $16 \mathrm{~S}$ and ITS Amplicon Sequencing. BioRxiv, 081257.

Ewing, L.L., Desjardins, C., Irby, D.C., Robaire, B., 1977. Synergistic interaction of testosterone and estradiol inhibits permatogensis in rats. Nature 269, 409-410.

Goeppert, N., Dror, I., Berkowitz, B., 2014. Detection, fate and transport of estrogen family hormones in soil. Chemosphere 95, 336-345.

Havens, S.M., Hedman, C.J., Hemming, J.D.C., Mieritz, M.G., Shafer, M.M., Schauer, J.J., 2020. Occurrence of estrogens, androgens and progestogens and estrogenic activity in surface water runoff from beef and dairy manure amended crop 
fields. Sci. Total Environ. 710, 136247.

He, E., Qiu, R., Cao, X., Song, L., Peijnenburg, W., Oiu, H., 2020. Elucidating toxicodynamic differences at the molecular scale between $\mathrm{ZnO}$ nanoparticles and $\mathrm{ZnCl}_{2}$ in Enchytraeus crypticus via nontargeted metabolomics. Environ. Sci. Technol. 54, 3487-3498.

Jarvinen, E., Kidron, H., Finel, M., 2020. Human efflux transport of testosterone, epitestosterone and other androgen glucuronides. J. Steroid Biochem. 197, 105518.

Kolpin, D.W., Furlong, E.T. Meyer, M.T., Thurman, E.M., Zaugg, S.D., Barber, L.B., Buxton, H.T., 2002. Pharmaceuticals, hormones, and other organic wastewater contaminants in US streams, 1999-2000: a national reconnaissance. Environ. Sci. Technol. 36, 1202-1211.

Li, X., Ban, Z., Yu, F., Hao, W., Hu, X., 2020. Untargeted metabolic pathway analysis as an effective strategy to connect various nanoparticle properties to nanoparticleinduced ecotoxicity. Environ. Sci. Technol. 54, 3395-3406.

Li, X.N., Qu, C.S., Bian, Y.R., Gu, C.G., Jiang, X., Song, Y., 2019. New insights into the responses of soil microorganisms to polycyclic aromatic hydrocarbon stress by combining enzyme activity and sequencing analysis with metabolomics. Environ. Pollut. 255, 9.

Ma, R., Zhang, T.C., Bartelt-Hunt, S.L., Oi, Y., Kranz, W.L., Snow, D.D., Mader, T.L., Shapiro, C.A., Shelton, D.P., van Donk, S.J., Tarkalson, D.D., Ensley, S., 2015. Influence of soil properties and test conditions on sorption and desorption of testosterone. J. Environ. Eng. 141, 04015006.

Macey, M.C., Pratscher, J., Crombie, A.T., Murrell, J.C., 2020. Impact of plants on the diversity and activity of methylotrophs in soil. Microbiome 8, 17.

Moreira, A., Moraes, L.A.C., 2017. Yield, nutritional status and soil fertility cultivated with common bean in response to amino-acids foliar application. J. Plant Nutr. $40,344-351$

Pagano, L., Serving, A.D., De La Torre-Roche, R., Mukherjee, A., Majumdar, S., Hawthorne, J., Marmiroli, M., Maestri, E., Marra, R.E., Isch, S.M., Dhankher, O.P., White, J.C., Marmiroli, N., 2016. Molecular response of crop plants to engineered nanomaterials. Environ. Sci. Technol. 50, 7198-7207.

Qi, Y., Zhang, T.C., 2016a. Sorption of testosterone on partially-dispersed soil particles of different size fractions: Methodology and implications. Water Res. 92,
$1-10$.

Qi, Y., Zhang, T.C., 2016b. Transport of manure-borne testosterone in soils affected by artificial rainfall events. Water Res. 93, 265-275.

Quideau, S.A., McIntosh, A.C.S., Norris, C.E., Lloret, E., Swallow, M.J.B., Hannam, K. 2016. Extraction and analysis of microbial phospholipid fatty acids in soils. JoveJ. Vis. Exp. 116, 54360.

Ragot, S.A., Kertesz, M.A., Bunemann, E.K., 2015. phoD alkaline phosphatase gene diversity in Soil. Appl. Environ. Microbiol. 81, 7281-7289.

Ruan, A., Zong, F., Zhao, Y., Liu, C., Chen, J., 2014. Effects of 17 beta-estradiol pollution on water microbial methane oxidation activity. Environ. Toxicol. Chem. 33, $768-775$.

Stumpe, B., Marschner, B., 2007. Long-term sewage sludge application and wastewater irrigation on the mineralization and sorption of 17 beta-estradiol and testosterone in soils. Sci. Total Environ. 374, 282-291.

Vendl, C., Ferrari, B.C., Thomas, T., Slavich, E., Zhang, E., Nelson, T., Rogers, T., 2019. Interannual comparison of core taxa and community composition of the blow microbiota from East Australian humpback whales. FEMS Microbiol. Ecol. 95.

Withers, E., Hill, P.W., Chadwick, D.R., Jones, D.L., 2020. Use of untargeted metabolomics for assessing soil quality and microbial function. Soil Biol. Biochem. 143, 107758.

Xia, J., Wishart, D.S., 2010. MSEA: a web-based tool to identify biologically meaningful patterns in quantitative metabolomic data. Nucleic Acids Res. 38 , W71-W77.

Ye, D.H., Li, T.X., Yu, H.Y., Zou, L.K., Huang, H.G., Zhang, X.Z., Liu, J.B., 2020. Characteristics of bacterial community in root-associated soils of the mining ecotype of Polygonum hydropiper, a P-accumulating herb. Appl. Soil Ecol. 150, 8.

Zhang, H., Huang, M., Zhang, W., Gardea-Torresdey, J.L., White, J.C., Ji, R., Zhao, L. 2020. Silver nanoparticles alter soil microbial community compositions and metabolite profiles in unplanted and cucumber-planted soils. Environ. Sci. Technol. 54, 3334-3342.

Zhao, L., Zhang, H., White, J.C., Chen, X., Li, H., Qu, X., Ji, R., 2019. Metabolomics reveals that engineered nanomaterial exposure in soil alters both soil rhizosphere metabolite profiles and maize metabolic pathways. Environ. Sci. Nano 6 1716-1727. 